Thorax (1969), 24, 585.

\title{
Mediastinoscopy: A clinical evaluation of 400 consecutive cases
}

\author{
C. L. SARIN ${ }^{1}$ AND H.C. NOHL-OSER \\ From the Thoracic Surgical Unit, Harefield Hospital, Harefield, Middlesex
}

\begin{abstract}
Mediastinoscopy was carried out in 400 cases, including 296 of bronchogenic carcinoma. At the time of presentation the new growth had already spread to involve the mediastinal lymph nodes in slightly more than $50 \%$ of these. The incidence of involvement was $76 \%$ in oat-cell and $35 \%$ in squamous-cell carcinoma. Non-resectability at thoracotomy was encountered in seven out of 120 patients. We advocate this procedure in every case of bronchogenic carcinoma which is considered operable on other counts. In patients in whom the mediastinal lymph nodes are invaded by growth we prefer radical radiotherapy to surgery, as the long-term survival of the two methods is comparable. This procedure may be the only source of positive histological proof of diagnosis, not only in carcinoma but in other types of intrathoracic disease. We believe that this procedure reduces the number of unnecessary exploratory thoracotomies.
\end{abstract}

Carlens (1959) introduced diagnostic exploration of the superior mediastinum. The space explored is part of the superior mediastinum which is situated around the intrathoracic part of the trachea, its bifurcation, and the proximal part of the bronchi. This investigation has been generally accepted on the continent of Europe as being useful in the diagnosis of intrathoracic disease and to assess the spread of carcinoma of the bronchus; but it has been used less frequently in Great Britain. We have been using mediastinoscopy since March 1963. Four hundred examinations have been carried out up to June 1968. The purpose of this study is to analyse the results and usefulness of these examinations.

\section{SURGICAL TECHNIQUE}

The technique used for this investigation has already been described by one of us (Nohl-Oser, 1965). The usefulness of this investigation is that it allows palpation as well as inspection of the lymph nodes and tissues around the trachea and its bifurcation. Mobility or fixity of the tissues can be assessed. It has also been valuable in the preoperative assessment of Pancoast tumours, as invasion of the vertebrae, when present, can be appreciated. In the majority of cases a biopsy for histological examination is obtained.

A short, fat neck or a rigid, anthritic cervical spine may make the operation difficult or even preclude the introduction of the mediastinoscope. In some cases finger palpation of the tissues may be all that is pos-

'Present address: Cardiothoracic Unit, St. Thomas' Hospital, London, S.E.1 sible. Biopsy in such cases can be obtained from tissues just inside the thoracic inlet. Bleeding, in the presence of incipient or developed superior vena caval obstruction, or dense fibrosis of the pre-tracheal fascia, can make the procedure difficult or impossible. Adherence of the lymph nodes or malignant tissues to the major blood vessels or nerves, especially the recurrent laryngeal nerve, calls for caution when taking a biopsy. In this series we were unable to introduce the mediastinoscope on four occasions, that is a failure rate of $1 \%$. In two cases during our first 50 examinations major bleeding, which needed thoracotomy, occurred. Details of this have been described elsewhere (Nohl-Oser, 1965). In the last 350 examinations no morbidity or mortality has been recorded.

\section{CLINICAL MATERIAL}

Cases in which mediastinoscopy was carried out can be divided into two categories: (a) bronchogenic carcinoma, where it was used to assess the extent and location of the spread of the neoplasm and in some cases to establish the diagnosis when bronchoscopic examination did not provide biopsy material; and (b) other intrathoracic disease where diagnosis of an unidentified mediastinal mass or lung shadow was required.

The final diagnoses for which this procedure was carried out are listed in Table I.

BRONCHOGENIC CARCINOMA Two hundred and ninetysix patients suffering from bronchogenic carcinoma were examined. There were 257 men and 39 women, giving an approximate sex ratio of $6: 1$, which is the same as the figures issued by the Registrar General for bronchogenic carcinoma for 1965. The age incidence in this series is shown in the Figure. Seventy-six 
T A B L E I

DIAGNOSES IN 400 PATIENTS WHO UNDERWENT MEDIASTINOSCOPIC EXAMINATION

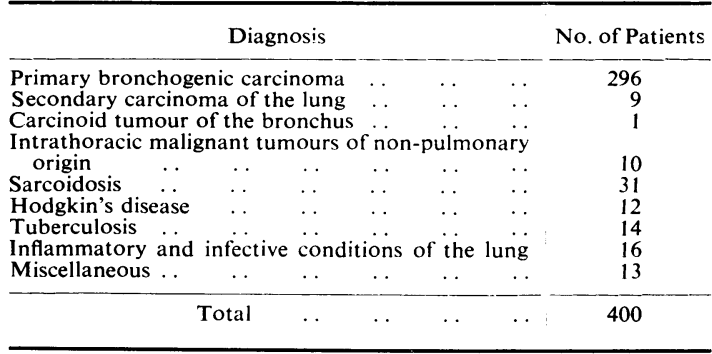

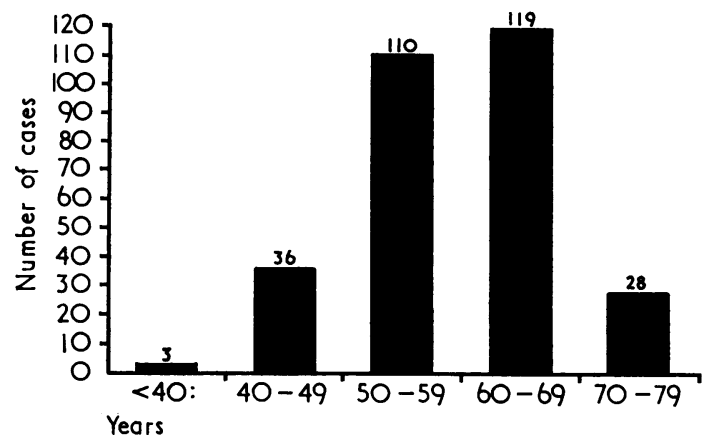

FIGURE. Age incidence in 296 cases of bronchogenic carcinoma examined mediastinoscopically.

per cent of the cases occurred in the age group 50 to 69. Only three patients were under 40 years of age. In 149 patients the modiastinal nodes or tissues were considered to be malignant. The histological types of tumour found and the incidence of mediastinal involvement in each type are shown in Table II. Malignant nodes can usually be recognized at mediastinoscopy after opening the pretracheal fascia, a search being made for them in the right and left paratracheal, in the tracheo-bronchial angles, and in the sub-carinal regions. Mobility or fixity of these glands is assessed by palpation. The region is then inspected with the help of the madiastinoscope. Suspicious nodes are dissected off vital structures and can be removed completely or a biopsy can be taken.

T A B L E I I

HISTOLOGICAL TYPES OF BRONCHOGENIC CARCINOMA AND INCIDENCE OF MEDIASTINAL LYMPH NODE INVOLVEMENT

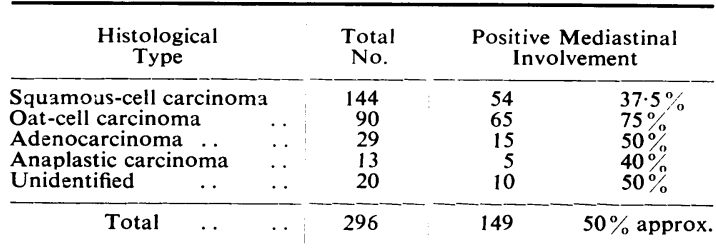

Should any node be inseparably adherent to a vita응 structure which might be damaged, a biopsy is no는 done. Bilateral involvement of the lymph nodes was seen in 12 patients, and contralateral involvemen without any ipsilateral involvement was seen in fiven In 14 patients biopsy was considered too dangerous? due to adherence and closeness of the nodes to the blood vessels or nerves, although they were considered to be involved on palpation. This leaves 135 patientf in whom a biopsy was obtained. In nine, histopatho logical proof of invasion was not obtained in spite of the presence of hard, fixed palpable nodes which at the time were deemed to be malignant. Thirty-severt patients in this group were considered inoperable oris account of the bronchoscopic findings, while in severv there was also evidence of distant spread.

In 147 patients where mediastinal nodes and tissues were found to be free of tumour invasion, two diects before surgery could be undertaken; necropsy showe $\mathbb{B}$ the cause of death in both to be myocardial infarction $\mathbb{\mathbb { D }}$ and the mediastinal nodes were free of growth. Fifteer, patients were found to be unfit for surgery becausæd of poor respiratory reserve. Evidence of contralatera $L$ and/or distant spread was present in another ten\& One hundred and twenty patients were submitted to6 thoracotomy, and in only seven could the tumour not be removed. There were three other patients with poof respiratory reserve in whom the tumour was not excised because a pneumonectomy rather than lobectomy would have been necessary. Thus $11 \%$ patients had a lung resection and in these mediastina $B$ node involvement was found in only nine cases. These should have been accessible at mediastinoscopy.

SECONDARY EXTRATHORACIC CARCINOMA There were seven such patients. The primary site was the breast: kidney, stomach, uterus, pancreas, and colon. An un known primary gave rise to multiple secondaries in both lungs. Mediastinal biopsy was positive in the first three but was negative in the other four.

OTHER THORACIC MALIGNANCIES There were 11 patient: suffering from other thoracic malignancies. In two with pleural mesothelioma, the lymph nodes and the mediastinal glands were found to be free of growth? The mediastinal glands were also found to be norma in a patient suffering from a carcinoid tumour where the diagnosis was established at thoracotomy. In severn cases the thymic region was involved and it was possible to cbtain a diagnostic biopsy by mediastinoscopto in only two of these. In the others a mass occupying the anterior mediastinum was palpable in front of the great vessels and a subsequent anterior mediastino $<$ tomy, by resecting the second costal cartilage, wa necessary to obtain a diagnostic biopsy. The histology in those seven involving the thymic region was three reticulum-cell sarcoma, three malignant lymphoma, and one squamous-cell carcinoma. In one patient whos had an oesophageal carcinoma the lymph nodes were infiltrated by growth.

SARCOIDOSIS Thirty-one patients, suspected cases of sarcoidosis, were referred to us by the physicians for 
mediastinal lymph node biopsy. They usually had radiological evidence of mediastinal lymph node enlargement or vague peripheral or hilar lung shadows. We were able to confirm the diagnosis in 24 . The mediastinal lymph nodes in sarcoidosis are usually large and fleshy. They are found in the paratracheal regions or superior tracheo-bronchial angles. Usually they are multiple and only exceptionally is a single node seen. In long-standing disease there is frequently evidence of fibrosis in the paratracheal regions, making access to the nodes more difficult. One of these patients with sarcoidosis had a shadow in the posterior mediastinum associated with mediastinal enlargement on the chest radiograph. One would normally associate this appearance with a primary lung carcinoma with mediastinal node involvement, but biopsy of the mediastinal glands showed sarcoidosis and the posterior mediastinal shadow proved to be a neurofibroma at subsequent thoracotomy.

TUBERCULOSIS In 14 patients who had pulmonary tuberculosis the usual investigations were found to be unhelpful and mediastinoscopy was carried out. The mediastinal lymph nodes were submitted for culture and sensitivity in addition to histological examination. Nine out of the 14 proved to be tuberculous. In five of these Mycobacterium tuberculosis was cultured. Of the five negative cases, two were proved to be of old healed tuberculosis at thoracotomy. In two, large firm nodes were seen and felt on mediastinoscopic examination but histological confirmation was not obtained. The procedure could not be completed in one because of a short, fat neck.

HODGKIN'S DISEASE In 12 patients with Hodgkin's disease mediastinoscopy was carried out. Enlarged, firm, fleshy nodes were felt along the trachea and in the tracheo-bronchial angles, sometimes extending up to the thoracic inlet. In 10 , histological examination of the nodes proved the diagnosis. In two, enlarged masses of lymph nodes could be felt in the anterior mediastinum, i.e., anterior to the great vessels. In these patients material for biopsy was safely obtained by resection of the second costal cartilage.

Miscellaneous Conditions Twenty-nine patients were submitted to mediastinoscopic examination for varied conditions such as unilateral diaphragmatic elevation or an undiagnosed atypical lung shadow with or without mediastinal enlargement. These are listed in Table III.

\section{T A B L E I I I}

MISCELLANEOUS NON-MALIGNANT CONDITIONS FOR WHICH MEDIASTINOSCOPY WAS CARRIED OUT

\footnotetext{
Inflammatory condition of lung

Calcified goitre pressing on trachea and causing

recurrent infection

Pericardial cyst

Talc pneumoconiosis

Idiopathic phrenic nerve palsy

Amyloid disease of the lung

Hamman Rich syndrome

Hamman Rich syndrome $\ldots$... ...

pressure and infection

Undiagnosed
}

16

1

1

The biggest single group was unresolved or slowly resolving pneumonitis causing a radiological shadow. Mediastinoscopy was carried out to exclude malignancy. The value of this examination lay in negative reports.

One patient who had a symptomless pericardial cyst causing a mediastinal shadow deserves special mention. On mediastinoscopic examination $150 \mathrm{ml}$. of liquid was aspirated. The procedure in this case, besides being diagnostic, was therapeutic.

\section{DISCUSSION}

In 1949 Daniels introduced the technique of scalene node or fat biopsy. In $7 \%$ of cases of apparently operable bronchogenic carcinoma, where the scalene nodes were not palpable, the removed tissue showed malignant infiltration. Since the lymphatic spread of carcinoma usually takes place in a progressive manner from the site of the primary to the root of the lung and from there mainly upwards through the mediastinal nodes to the scalene nodes, Carlens mediastinoscopy was a natural extension of Daniels scalene node biopsy.

Initially this investigation was used by us only when bronchogenic carcinoma was associated with radiological or clinical evidence of mediastinal spread or in other conditions described elsewhere. Gradually its use has been extended and for the past two years it has been a part of the preoperative assessment in all cases of bronchogenic carcinoma. In nearly $50 \%$ of all cases of bronchogenic carcinoma thus examined, mediastinal lymph node involvement was found at the time of presentation. Other authors describe a range of $23 \%$ to $46 \%$. One of us (Nohl, 1962) dissected 211 surgical specimens and found mediastinal lymph node involvement in $34 \%$ of squamous carcinoma and in $60 \%$ of undifferentiated carcinoma. Pearson (1968), in a recent study from Toronto General Hospital, reported an incidence of $33 \%$ in apparently operable cases. However, out of 149 patients in this series in whom mediastinal gland involvement was demonstrated, 37 were also considered to be inoperable from the bronchoscopic point of view and seven had evidence of distant spread. All in whom mediastinal lymph nodes were found to be involved were treated with radiotherapy in preference to surgery. A few of these lesions could have been resected, but we believe that the results of surgery in patients who have mediastinal node involvement are no better than those obtained by radical radiotherapy.

Nohl (1962) and Bærgh and Scherstén (1965) have shown that in bronchogenic carcinoma with invasion of the mediastinal lymph nodes four-year survival is less than $9 \%$ after 
surgical therapy. Morrison and Deeley (1960) showed that when inoperable bronchogenic carcinomata were treated with radical radiotherapy the four-year survival rate was $6 \%$. Given that surgery carries a mortality of $5 \%$ to $10 \%$, any marginal surgical advantage over radiotherapy is nullified. Nearly half of these cases are of the oat-cell variety, for which surgery and radiotherapy are of equal value (Morrison, Deeley, and Cleland, 1963).

Mediastinoscopy also reduces the number of patients in whom resection is not possible at thoracotomy. Radinov (1962) analysed data (nearly 50,000 cases reported by 87 surgeons) and described the non-resectability rate as $43 \%$. Reynders (1964), in a review of the literature, described the range of non-resectability as between $20 \%$ and $50 \%$ and in his personal series $40 \%$. In $86 \%$ of the non-resectable lesions mediastinal extension was the cause of non-resectability. Following the use of mediastinoscopy Reynders reduced his non-resectability figures at thoracotomy from $40 \%$ to $10 \%$. Pearson (1968) reduced his from $23 \%$ to $6 \%$. In this series the non-resectability rate after the introduction of mediastinoscopy has been reduced to $6 \%$ ( 7 out of 120 ). Even to those surgeons who advocate radical surgery mediastinoscopy can provide information as to the site and extent of spread which may be of help in planning surgery. In this series bilateral involvement of the lymph nodes was demonstrated in 12 patients and contralateral involvement without ipsilateral invasion in five. These patients are beyond surgical help.

In some cases a solitary metastasis in the lung from a primary growth elsewhere in the body may b? profitably resected, but we think that mediast:noscopy may be one of the only means of proving that an apparently single metastasis is not in fact a solitary secondary.

In miscellaneous conditions like sarcoidosis, Hodgkin's disease, and tuberculosis, mediastinoscopy has frequently been helpful when simpler diagnostic techniques have failed to establish the diagnosis, and thus exploratory thoracotomies were avo:ded.
Mediastinoscopy was carried out in 400 case including 296 cases of bronchogenic carcinoma At the time of presentation the new growth has already spread to involve the mediastinal lymph nodes in slightly more than $50 \%$ of cases. The incidence of involvement was $76 \%$ in oat-cell and $35 \%$ in squamous-cell carcinoma. Non-resectability at thoracotomy was encountered in seveow out of 120 cases. We advocate this procedure i⿱ every case of bronchogenic carcinoma which $\vec{x}$ considered operable on other counts. In patients in whom the mediastinal lymph nodes are invadedh by growth, we prefer radical radiotherapy to surgery, as the long-term survival of the two methods is comparable.

This procedure can be the only source of positive histological proof of diagnosis not only in carcinoma but in other types of intrathoract disease. We balieve that this procedure reduces the number of unnecessary exploratory thoracotomies.

The authors would like to record their gratefu्f thanks to Miss Ponter, Medical Records Office? Hillingdon Hospital, Uxbridge, and to Mrs. Fro Medical Records Officer, Harefield Hospital ; to Mr롱 S. Sarin for help in the statistical analysis; and Mrs. P. Barsby and Miss P. Healey for secretari help.

\section{REFERENCES}

Bergh, N. P., and Scherstén, T. (1965). Bronchogenic carcinoma. $\overline{\mathrm{N}}$ follow-up study of a surgically treated series with special referen to the prognostic significance of lymph node metastases. Acta chir. scand., suppl. 347.

Carlens, E. (1959). Mediastinoscopy: a method for inspection an tissue biopsy in the superior mediastinum. Dis. Chest, 36, 343.

Daniels, A. C. (1949). A method of biopsy useful in diagnosing certa intrathoracic diseases. Ibid., 16, 360 .

Morrison, R., and Deeley, T. J. (1960). Inoperable cancer of the brom chus treated by megavoltage X-ray therapy. Lancet, $2,618$. and Cleland, W. P. (1963). The treatment of carcinoma 8 the bronchus. A clinical trial to compare surgery and supervoltage radiotherapy. Ibid., 1, 683. Nohl, H. C. (1962). The Spread of Carcinoma of the Bronchus. Lloy@
Luke, London.

Nohl-Oser, H. C. (1965). Mediastinoscopy. Brit. med. J., 1, 1167. D

Pearson, F. G. (1968). An evaluation of mediastinoscopy in the management of presumably operable bronchial carcinoma. $F$ thorac. cardiovasc. Surg., 55, 617.

Radinov (1962). Quoted by Jepsen, O., Mediastinoscopy, 1966. Einar Munskgaard, Copenhagen. Reynders, H. (1964). Mediastinoscopy in bronch ogenic cancer. $D$ Chest, 45. 606.
Cos 